\title{
Selective synchronization of coupled bifurcating neurons for phase shift of background oscillation
}

\author{
Akihiro Yamaguchi*, Yutaka Yamaguti \\ Department of Information and Systems Engineering, Fukuoka Institute of Technology, \\ 3-30-1 Wajiro-higashi, Higashi-ku, Fukuoka, 811-0295, Japan \\ Masao Kubo \\ Department of Computer Science, National Defense Academy of Japan, \\ 1-10-20 Hashirimizu, Yokosuka, Kanagawa, 239-8686, Japan \\ E-mail: ${ }^{*} a k i @ f i t . a c . j p$
}

\begin{abstract}
Synchronization in coupled bifurcating neurons was studied from the view point of the selective formation of cell assembly. The bifurcating neuron is a simple chaotic neuron that exhibits chaotic inter-spike interval dynamics via the addition of sinusoidal background oscillation. In this research, we introduced the phase shift of the background oscillation and several types of phase response to the input spike sequence. Sixteen coupled bifurcating neurons with complete bidirectional coupling were numerically simulated and their synchronized behaviors were analyzed. As a result, the formation of chaotically synchronized cell assemblies for the phase shift value were observed.
\end{abstract}

Keywords: chaotic synchronization, neural coding, bifurcating neuron, binding information

\section{Introduction}

Synchronized neural activity has an important role in neural information coding and brain information processing ${ }^{1}$. The correlated firing among neurons has been observed in visual information processing in the brain $^{2,3}$. The authors have studied the chaotic synchronization of the neural spike response and its application to the segmentation of input images ${ }^{4}$; feature linking of the visual image of moving objects ${ }^{5}$; and the decomposition of superimposed chaotic spike sequences ${ }^{6}$. In order to represent multiple information by chaotic synchronized cell assemblies, confirming the coexistence of synchronized assemblies is necessary.

In this study, we constructed the network of the bifurcating neuron ${ }^{7}$ with complete bidirectional coupling and designed a coupling model to achieve selective chaotic synchronization based on the phase response. In Sections 2 and 3, we explain the bifurcating neuron network and coupling model. In Section 4, we describe the numerical simulation that is performed to examine the selective synchronization for the phase shift of the background oscillation. We summarize the results in Section 5.

\section{Network model of the bifurcating neuron}

The bifurcating neuron was introduced by Lee and Farhat ${ }^{7}$. In our previous research ${ }^{6}$, we defined the bifurcating neuron as a form of the spike response model ${ }^{8}$ to extend the coupling term based on the phase response.

In the following sections, we explain the extended bifurcating neuron model and its network. Let the network of bifurcating neurons consist of $N$ neurons, where $n^{(i)}$ is the $i$-th neuron. The dynamics of the $i$-th bifurcating neuron is defined as follows:

$$
\begin{gathered}
u^{(i)}(t)=u_{\text {rest }}+\eta^{(i)}(t)+\xi_{+}^{(i)}(t)+ \\
\xi_{-}^{(i)}(t)+v^{(i)},
\end{gathered}
$$


where $u^{(i)}, u_{\text {rest }}, \eta^{(i)}, \xi_{+}^{(i)}, \xi_{-}^{(i)}$, and $v^{(i)}$ denote the internal potential of $n^{(i)}$, resting potential, kernel function of internal dynamics, positive coupling term, negative coupling term, and external noise, respectively. The coupling terms are described in the next section.

The kernel function $\eta^{(i)}$ is defined as

$$
\begin{gathered}
\eta^{(i)}(t)=\alpha\left(t-t_{\text {last }}^{(i)}\right)+\eta_{0}\left(t_{\text {last }}^{(i)}, \phi^{(i)}\right) ; \\
\eta_{0}(t, \phi)=A_{\eta} \sin (2 \pi \omega t+\phi),
\end{gathered}
$$

where $\alpha$ is the time constant of the kernel $\eta^{(i)}$, and $t_{\text {last }}^{(i)}$ is the last firing time of $n^{(i)}$. The internal potential $u^{(i)}$ is linearly increasing by the $\operatorname{kernel} \eta^{(i)}$. The neuron $n^{(i)}$ is firing when $u^{(i)}$ exceeds the threshold, $\theta$, and then $u^{(i)}$ is reset to the initial potential given by the background oscillation, $\eta_{0}(t, \phi)$. The constants, $A_{\eta}, \omega$, and $\phi^{(i)}$ are the amplitude, frequency, and phase shift value of background oscillation, respectively.

The dynamics of a single neuron without the coupling term is the same as with the original bifurcating neuron. The single neuron without coupling exhibits various

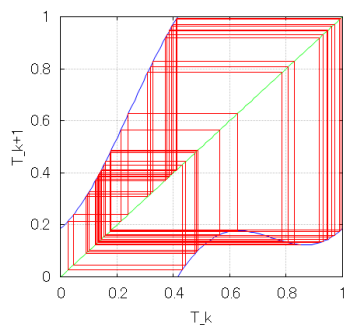

(a)

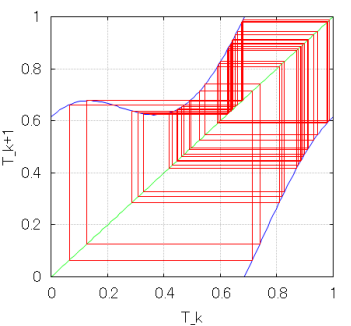

(b)
Fig. 1. Examples of the chaotic one dimensional map of the phase of a single bifurcating neuron with the phase shift (a) $\phi=$ $\pi / 2$ and (b) $\phi=3 \pi / 2$.
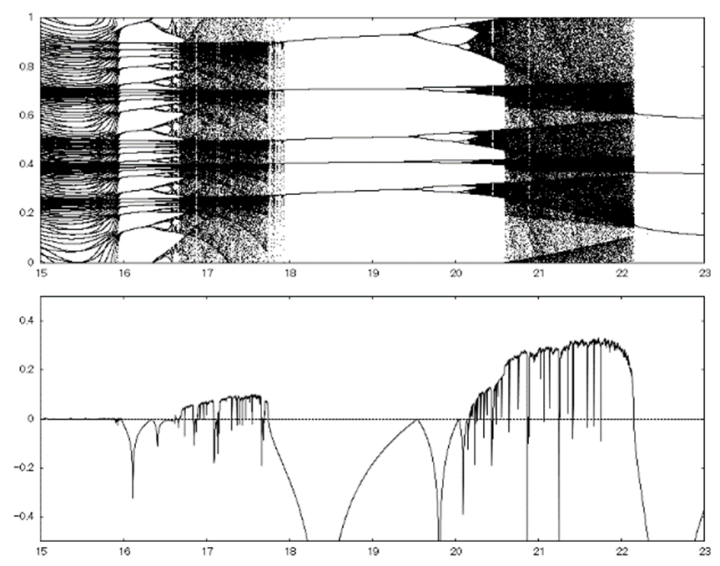

Fig. 2. The bifurcation diagram and Lyapunov exponents of a single bifurcating neuron without coupling. chaotic dynamics in the inter-spike interval, and the phase of the next firing time is determined by the chaotic one dimensional map of the phase of the last firing time $e^{6,7}$. The shape of this chaotic map is determined by the phase shift value, $\phi^{(i)}$. Examples of this chaotic map are shown in Fig. 1. Furthermore, the bifurcation diagram and Lyapunov exponents of this map are shown in Fig. 2.

\section{Coupling model with phase response}

Let the set $S^{(k)}=\left\{t_{0}^{(k)}, t_{1}^{(k)}, t_{2}^{(k)}, \cdots\right\}$ be a set of firing time of the neuron, $n^{(k)}$, where $t_{j}^{(k)}$ is the firing time of $j$-th spike of $n^{(k)}$. The set firing time of the input spikes to $n^{(i)}$ is denoted as $\Gamma^{(i)}$. For bidirectional complete coupling, $\Gamma^{(i)}$ is defined as

$$
\Gamma^{(i)}=\bigcup_{0 \leq k \neq i<N} S^{(k)}
$$

The coupling term $\xi_{+}^{(i)}$ and $\xi_{-}^{(i)}$ in Eq. (1) are the summation of the positive and negative phase responses $\varepsilon_{+}^{(i)}$ and $\varepsilon_{-}^{(i)}$, respectively, such that

$$
\xi_{ \pm}^{(i)}(t)=\sum_{s \in \Gamma^{(i)}, t_{\text {last }}^{(i)} \leq s<t} \varepsilon_{ \pm}^{(i)}(s) .
$$

The simple phase response is a constant value, such that

$$
\varepsilon_{ \pm}^{(i)}(s)= \pm \beta_{ \pm},
$$

where $\beta_{+}$and $\beta_{-}$are non-negative coupling constants. The next firing time is hastened by the positive response and delayed by the negative response. We described coupling with these simple responses as constant positive and negative coupling, respectively.

We designed the phase response to strengthen the chaotic synchronization between coupled neurons with the same phase shift value ${ }^{6}$. The positive phase response based on the predicted next firing time is defined as

$$
\varepsilon_{+}^{(i)}(s)=\left\{\begin{array}{cc}
0 & s<\hat{t}_{\text {next }}^{(i)}-\Delta_{\varepsilon} \\
+\beta_{+} & \hat{t}_{\text {next }}^{(i)}-\Delta_{\varepsilon} \leq s<\hat{t}_{\text {next }}^{(i)} \\
0 & \hat{t}_{\text {next }}^{(i)} \leq s
\end{array}\right.
$$

where $\hat{t}_{\text {next }}^{(i)}$ is the predicted next firing time of $n^{(i)}$, such that

$$
\hat{t}_{\text {next }}^{(i)}=t+\frac{\theta-u(t)}{\alpha} .
$$


We describe this coupling as adaptive positive coupling. The negative phase response based on the last firing time is defined as

$$
\varepsilon_{-}^{(i)}(s)=\left\{\begin{array}{cc}
0 & s \leq t_{\text {last }}^{(i)} \\
-\beta_{-} \frac{s-t_{\text {last }}^{(i)}}{\Delta_{\varepsilon}} & t_{\text {last }}^{(i)}<s \leq t_{\text {last }}^{(i)}+\Delta_{\varepsilon} . \\
0 & t_{\text {last }}^{(i)}+\Delta_{\varepsilon}<s
\end{array}\right.
$$

We describe this coupling as adaptive negative coupling.

If time, $s$, is within the range, $\Delta_{\varepsilon}$, from the predicted next firing time $\hat{t}_{\text {next }}^{(i)}$, then the phase response is positive, so that the next firing time is hastened. On the other hand, if time, $s$, is within the range, $\Delta_{\varepsilon}$, from the last firing time, $t_{\text {last }}^{(i)}$, then the phase response is negative to delay the next firing time. By using the phase responses in Eq. (7) and (9), the decomposition of the superimposed chaotic spike sequences with the different phase shift values is possible ${ }^{6}$.

\section{Numerical experiments}

\subsection{Experimental setting}

We numerically simulated a network of 16 bifurcating neurons to analyze the selective synchronization to the same phase shift value for complete bidirectional coupling. We assigned 4 different phase shift values, as follows:

$$
\phi^{(i)}=\Delta_{\phi}\lfloor i / 4\rfloor(i=0, \cdots, 15),
$$

where $0<\Delta_{\phi} \leq \frac{\pi}{2}$ is the difference between the assigned phase shift values. In this setting, each 4 neurons have the same phase shift value. We examined the selective synchronization among each 4 neurons with the same phase shift value. Numerical simulation was performed using the following settings: $\alpha=100, \theta=-30$, $u_{\text {rest }}=-70, A_{\eta}=21.5, \omega=1, \beta_{-}=\beta_{+}=2.1, \Delta_{\varepsilon}=$ 0.05 , and $\Delta_{\phi}=\pi / 2$.

\subsection{Characteristics of synchronization}

As an index of synchronization between two spike sequences, the synchronization ratio was defined as

$$
\begin{gathered}
S R\left(S^{(i)} ; S^{(k)}\right)=\#\left\{t^{i}|| t^{i}-t^{k} \mid \leq \Delta_{S},\right. \\
\left.t^{i} \in S^{(i)}, t^{k} \in S^{(k)}\right\} / \# S^{(k)},
\end{gathered}
$$

where \# $(\cdot)$ denotes the number of elements and $\Delta_{S}$ is the temporal resolution of the firing time. Furthermore, we characterized the assembly formation of the synchronized cluster of neurons by defining the mean

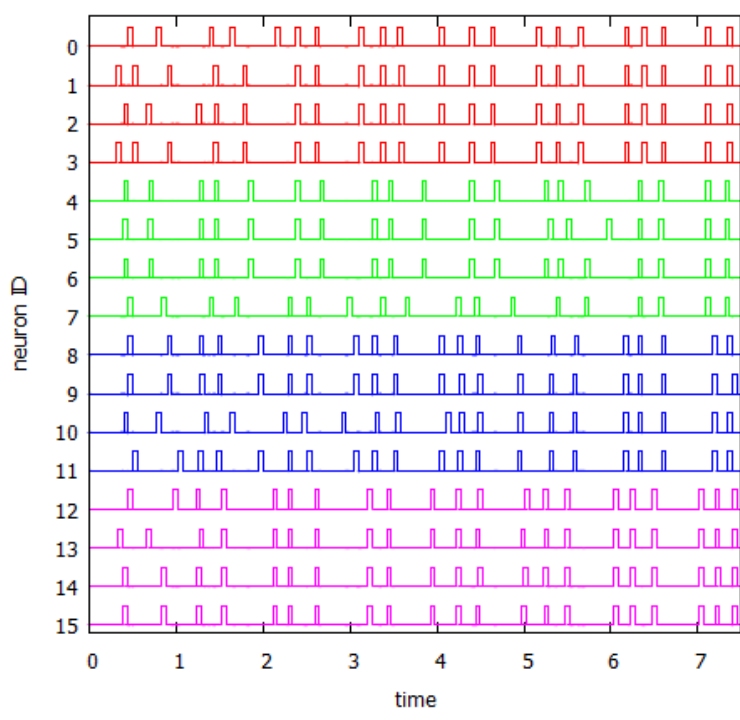

Fig. 3. An example of generated spike sequences for the network with the adaptive positive and negative coupling.

synchronization ratio for the neurons with the same phase shift value, such that

$$
M S R_{\phi^{(i)}=\phi^{(k)}}=\sum_{\substack{0 \leq i \neq k \leq 15, \phi^{(i)}=\phi^{(k)}}} S R\left(S^{(i)} ; S^{(k)}\right) / 48,
$$

and mean synchronization ratio for neurons with the different phase shift values, such that

$$
\operatorname{MSR}_{\phi^{(i)} \neq \phi^{(k)}}=\sum_{\substack{0 \leq i \neq k \leq 15, \phi^{(i)} \neq \phi^{(k)}}} S R\left(S^{(i)} ; S^{(k)}\right) / 192 .
$$

\subsection{Experimental results}

An example of our generated spike sequences from the numerical simulation are shown in Fig. 3, where the phase response coupling is the adaptive positive and negative coupling. The generated spikes were synchronized for each 4 neurons using the same phase shift value, and selective synchronization for the same phase shift value was observed. We numerically simulated the network and evaluated the synchronization ratio to analyze the effect of coupling, and the mean ratio for each of the 5 types of coupling that were defined in Section 3. Results are shown in Fig. 4 and Table 1. These results indicated that selective synchronization was observed for coupling types (c) and (e). The mean synchronization ratios were greater than $90 \%$ for the 


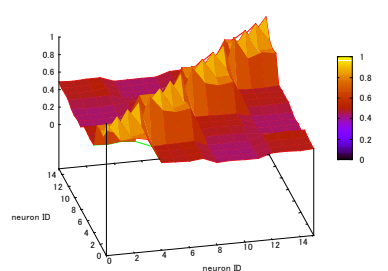

(a) Constant Positive

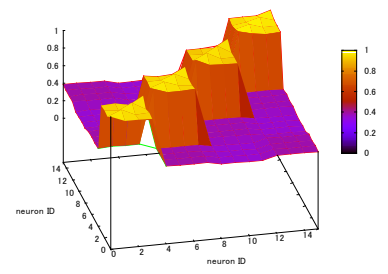

(c) Adaptive Positive

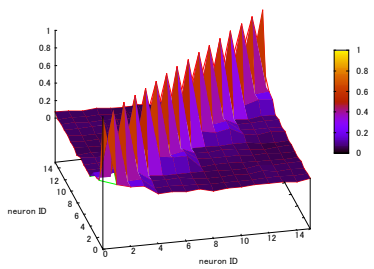

(b) Constant Negative

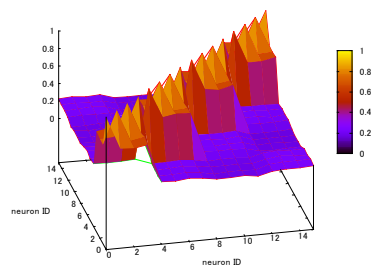

(d) Adaptive Negative

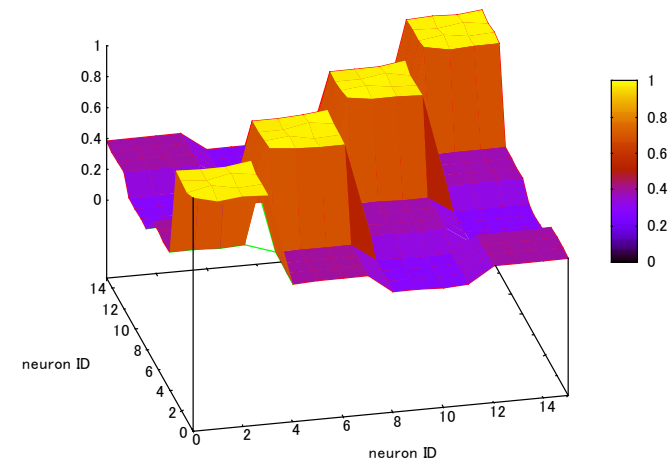

(e) Adaptive Positive and Negative (both)

Fig. 4. The synchronization ratio between two neurons for (a) constant positive, (b) constant negative, (c) adaptive positive, (d) adaptive negative, and (e) both adaptive positive and negative coupling types.

neurons with the same phase shift value, and less than $40 \%$ for the neurons with the different phase shift values.

\section{Conclusion}

We have constructed a bifurcating neuron network, and assessed the formation of chaotically synchronized cell assemblies for several types of coupling model. As a result, the chaotically synchronized cell assemblies were observed for adaptive positive coupling of the phase response, where neurons that have the same phase shift value were selectively synchronized. This result indicates the possibility of binding information based on the phase shift value of the background oscillation. Our future work will seek to analyze the effect of adaptive coupling using a different chaotic neuron model, which will further elucidate such information coding.
Table 1. Mean synchronization ratio for the neurons with the same phase shift value, $M S R_{\phi^{(i)}=\phi^{(k)}}$, and for the neurons with the different phase shift values, $M S R_{\phi^{(i)} \neq \phi^{(k)}}$.

\begin{tabular}{lcc}
\hline Coupling type & $M S R_{\phi^{(i)}=\phi^{(k)}}$ & $M S R_{\phi^{(i)} \neq \phi^{(k)}}$ \\
\hline \hline (a) Constant Positive & 0.7861 & 0.4766 \\
\hline (b) Constant Negative & 0.1819 & 0.0638 \\
\hline (c) Adaptive Positive & 0.9414 & 0.3694 \\
\hline $\begin{array}{l}\text { (d) Adaptive Negative } \\
\text { (e) Adaptive Positive } \\
\text { and Negative }\end{array}$ & 0.6520 & 0.2057 \\
\hline
\end{tabular}

\section{Acknowledgements}

This work was partially supported by MEXT KAKENHI Grant Number 15H05878 and 16K00409.

\section{References}

1. Fuji, H., Ito, H., Aihara, K., Ichinose, N., and Tsukada, M., Dynamical cell assembly hypothesis - Theoretical possibility of spatio-temporal coding in the cortex, Neural Networks, Vol.9, No.8, 1996, pp.1303-1350.

2. Gray, C.M., Koenig, P., Engel, A. K., and Singer, W., Oscillatory responses in cat visual cortex exhibit intercolumnar synchronization which reflects global stimulus properties, Nature, 338, 1989, pp.334-337.

3. Eckhorn, R., Reitboeck, H.J., Arndt, M., Dicke, P., Feature linking via stimulus-evoked oscillations: experimental results from cat visual cortex and functional implications from a network model, International Joint Conference on Neural Networks, 1989, pp. 723-730.

4. M. Fujiwara, A. Yamaguchi, and M. Kubo, Synchronized Response to Grayscale Image Inputs in Chaotic Cellular Neural Network, J. Robotics, Networking and Artificial Life, Vol.2, No.1, 2016, pp. 26-29.

5. A. Yamaguchi, S. Arakane, and M. Kubo, Feature Linking using Synchronized Responses in Chaotic Cellar Neural Networks for Visual Stimulus of Moving Objects, J. Robotics, Networking and Artificial Life, Vol.2, No.4, 2016, pp. 230-233.

6. A. Yamaguchi, Y. Yamaguti, and M. Kubo, Decomposition of superimposed chaotic spike sequences by using the bifurcating neuron, to appear in Advances in cognitive neurodynamics (VI), Springer, 2018.

7. Lee G., and Farhat, N.H., The Bifurcating Neuron Network 1, Neural Networks, Vol. 14, pp. 115-131, 2001.

8. Gerstner, W., and Kistler, W., Spiking Neuron Models: Single Neurons Populations Plasticity, Cambridge University Press, 2002. 